\title{
Kompetenz und Vertrauen
}

Matthias Scholer

Wissenschaftsjournalist

\author{
PROFILES, EPAs, CanMEDS, Blended learning, CBME - die ärztliche Aus-, Weiter- und \\ Fortbildung befindet sich weltweit in einem tiefgreifenden Umbruch. Am dritten \\ Symposium für ärztliche Weiterbildner erhielten die zahlreichen Teilnehmenden \\ wertvolle Einblicke in neue Lehrmethoden und deren Umsetzung in Schweizer \\ Kliniken.
}

«Without teaching, an institution is not first class.» Mit diesem Zitat des Arztes William Osler eröffnete Jürg Hodler das diesjährige Symposium für ärztliche Weiterbildung. Hodler schilderte aus seiner Sicht als Ärztlicher Direktor des UniversitätsSpitals Zürich, wie wichtig eine zeitgemässe, praxisorientierte Weiterbildung junger Assistenzärzte für eine Institution ist. So spielen heutzutage bei der Stellenwahl junger Medizinerinnen und Mediziner Faktoren wie Angebot und Qualität der

\section{«Without teaching, an institution}

is not first class.»

Weiterbildung eine entscheidende Rolle - ein nicht zu vernachlässigender Punkt im Hinblick auf den chronischen Personalmangel. Jürg Hodler machte aber auch deutlich, dass man mit der Ausbildung junger Ärztinnen und Ärzte eine grosse Verantwortung übernimmt, Mehrkosten zu tragen hat, Fachwissen garantieren muss und genügend Energie braucht, um Weiterbildungsprogramme im Alltag konsistent durchzuziehen.

\section{Schrittweise Vertrauen aufbauen}

Die Ausbildner bei ihrer verantwortungsvollen Rolle zu stärken und ihnen neue Inputs auf den Weg zu geben, sei das Ziel dieser Veranstaltung, erinnerte Hans Ulrich Bucher, der Delegierte für ärztliche Weiterbildung am USZ. Deshalb stehe dieses dritte Symposium ganz im Zeichen der Kompetenzbasierten Weiterbildung. Ein zentraler Baustein dieses Modells sind die «Entrustable Professional Activities» (EPAs). Mit dieser Abkürzung sei nicht etwa ein ehemaliges Warenhaus gemeint, scherzte Bucher, sondern ein zeitgemässes Konzept, welches verstärkt die starren Lernziele nicht nur in der Weiterbildung, sondern auch im Studium ersetzen wird. Mit EPAs werden Arbeitseinheiten bezeichnet, die sich explizit vom klinischen Alltag aus definieren und die schrittweise auf die Weiterzubildenden übertragen werden können immer unter der Voraussetzung, dass die dafür notwendigen Kompetenzen ausreichend vorhanden sind. Sobald eine junge Ärztin oder ein junger Arzt eine bestimmte Arbeit selbständig durchführen kann, sei das Stadium des «Entrustment», des Anvertrauens, erreicht, erklärte Bucher. Dank diesem Konzept erlangen die Weiterzubildenden also nicht nur schrittweise mehr Kompetenzen; sie kommen zudem in den Genuss einer individualisierten Einarbeitung in ihr Fach.

\section{Zeit alleine genügt nicht, Kompetenzen zu erlangen}

Jason R. Frank kennt sich mit dem Konzept der EPAs bestens aus. Der Notfallmediziner und Leiter der ärztlichen Weiterbildungsabteilung des Royal College of Physicians and Surgeons of Canada war als Hauptredner geladen. Für den Kanadier steht fest, dass das Ausbildungssystem für die meisten Berufe im Gesundheitsbereich veraltet ist. Bislang basiert die Weiterbildung eines Assistenzarztes auf einem mehrjährigen Rotationsplan, den er durchlaufen muss, um die Zulassung zur fachärztlichen Prüfung zu erhalten. Zwar wisse dann ein Kandidat, auf welchem Stock er im Jahr 2020

Häufig arbeiten Ausbildner und Auszubildender aufgrund chronischen Zeitmangels parallel zueinander.

die Toiletten benutzen wird, aber ob er in diesem Jahr auch eine Vorgesetzte oder einen Vorgesetzten bekommt, der ihn pflichtbewusst führt und ausbildet, stehe in den Sternen, bemerkte der Experte provokativ. 
So komme es unweigerlich zu grossen Variationen im Fachwissen zwischen den einzelnen Ausbildungsstellen. Das bisherige System birgt laut Jason R. Frank noch andere Nachteile. Häufig arbeiten Ausbildner und Auszubildender aufgrund chronischen Zeitmangels parallel zueinander. In den meisten Fällen treffen sie sich bloss zu Schichtbeginn und -ende. Damit fehlen Zeitfenster, in denen die erfahrene Ärztin den Assistenzarzt bei seiner Arbeit beobachten kann, um ihm anschliessend ein konstruktives Feedback zu geben. Untersuchungen in Kanada zeigen zudem, dass Lehreinheiten meist ad hoc zustande kommen. Zu guter Letzt habe das ‘alte` Ausbildungsmodell noch den Nachteil, dass die Beurteilung der Lernenden zum Ende jeder Rotation meist oberflächlich und damit mit geringer Aussagekraft sei. Genug gute Gründe für den Experten, etwas zu ändern.

\section{Stufenweise zum Erfolg}

An der medizinischen Fakultät der Universität Ottawa kommt seit über zehn Jahren die sogenannte «Competency-based Medical Education" (CBME) zum Einsatz. $\mathrm{Zu}$ Beginn mussten für jede Fachrichtung Ziele formuliert werden, welche ein Absolvent am Ende seines Weiterbildungsprogramms zu erreichen hat. Darauf basierend sequenzierten die Experten den Weg zu diesen Zielen. Konkret wurde jedes Weiterbildungsprogramm in vier Stufen eingeteilt - von der Einführung in die Fachrichtung, über deren Grundlagen, die nötigen Kernkompetenzen bis hin zum Übergang in den selbständigen Praxisalltag. Auf jeder dieser Stufen muss eine Kandidatin, ein Kandidat spezifische EPAs

\section{$\mathrm{Zu}$ Beginn mussten für jede Fachrichtung} Ziele formuliert werden, welche ein Absolvent am Ende seines Weiterbildungsprogramms zu erreichen hat.

und klar definierte Meilensteine erreichen, um im Ausbildungsprogramm weiterzukommen. Ein solcher Aufbau habe vier grosse Vorteile, erklärte Jason R. Frank. Erstens sei allen Beteiligten von Beginn weg klar, was sie voneinander erwarten dürfen, zweitens sei die Rolle der Fakultät definiert, drittens könne die Kompetenz der jungen Ärztinnen und Ärzte standardisiert verbessert werden, und viertens basiere die Bewertung der einzelnen Kandidaten auf einer breiten, nachvollziehbaren Datenmenge. Den letzten Punkt versinnbildlichte Jason R. Frank mit der Bildqualität einer Fotografie - je mehr Pixel ein Bild enthält, umso schärfer und detaillierter werde es. Im Falle der CBME seien die einzelnen Pixel die sehr häufigen, kurzen Einschätzun-

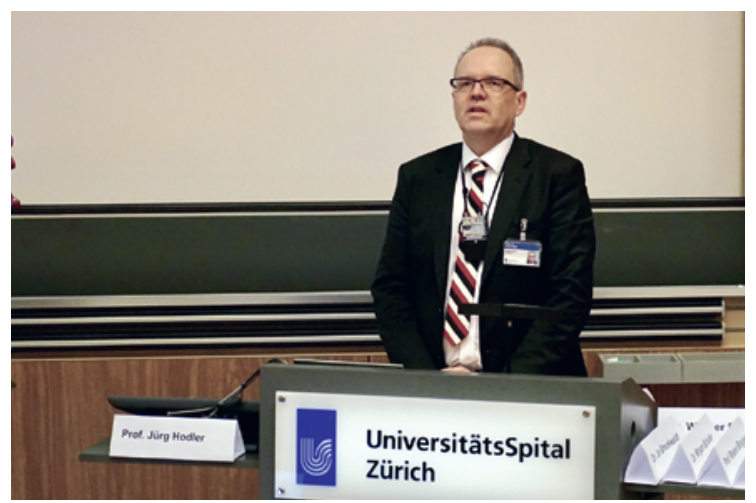

Jürg Hodler, Ärztlicher Direktor des USZ, eröffnete die Veranstaltung.

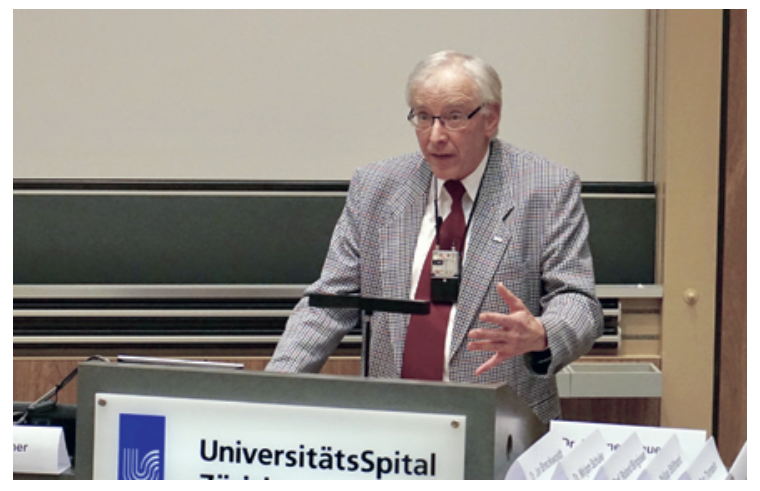

Organisator Hans Ulrich Bucher, der Delegierte für ärztliche Weiterbildung USZ.

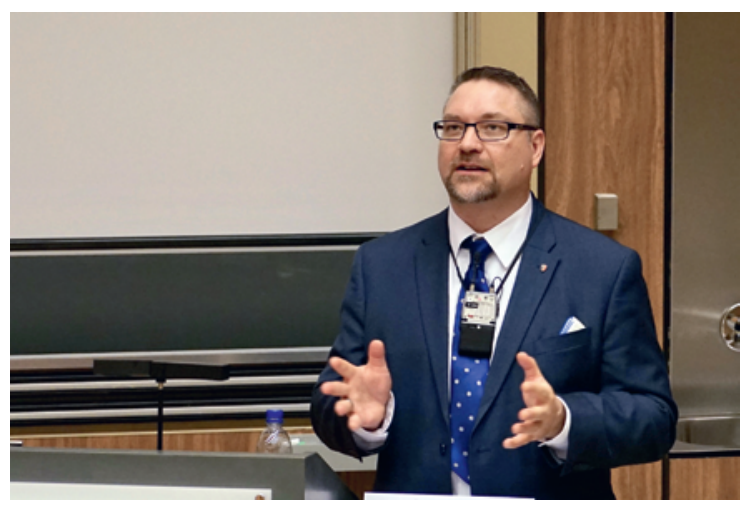

Hauptredner: Jason R. Frank, University of Ottawa.

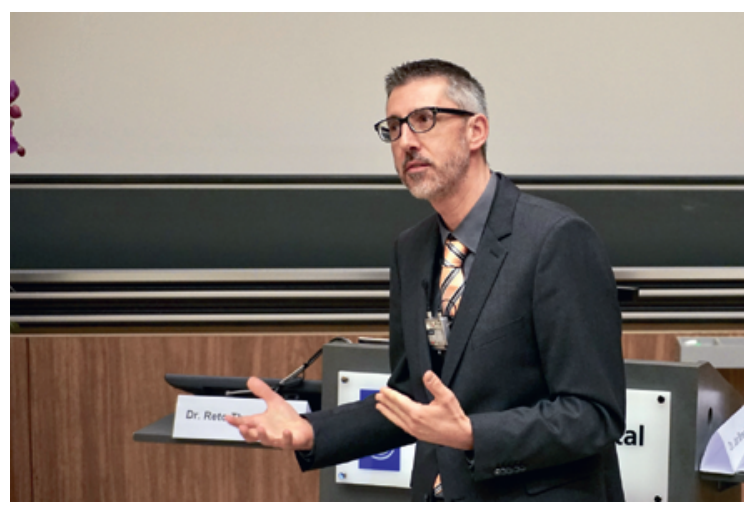

Reto Thomasin, Leitender Arzt Anästhesiologie, Kantonsspital Winterthur. 
gen jedes Kandidaten durch seine Vorgesetzten im elektronischen Kandidatendossier.

Die Fragen im Anschluss des Referates zeigten, dass die Implementierung neuer Weiterbildungsprogramme in den Schweizer Kliniken nicht reibungslos abläuft. Jason R. Frank ist sich dieser Schwierigkeit bewusst, und er zitierte eine englische Redewendung: «Changing a curriculum is like moving a graveyard.» Beides sei extrem schwierig, schliesslich hänge man an Dingen, auch wenn man wisse, dass sie längst tot sind.

\section{Umsetzung in der Schweiz}

Nach diesem vertieften Einblick in die Theorie und Praxis der CBME lag der Fokus während der zweiten Hälfte des Symposiums auf der Umsetzung moderner Weiterbildungsansätze hierzulande. Den Anfang machte dabei Reto Thomasin, Leitender Arzt am Institut für Anästhesiologie am Kantonsspital Winterthur. Nachdem an dieser Abteilung bereits seit längerer Zeit ein Logbuch und ein Testatheft zur Aufzeichnung der Fertigkeiten jedes Weiterzubildenden zum Einsatz kamen, wurden im vergangenen Jahr mehrere EPAs und Mei-

\section{Langfristiges Ziel der Winterthurer Anäs- thesisten sind eine Vernetzung mit anderen Weiterbildungsstätten und ein durchgehendes Curriculum.}

lensteine definiert, um insbesondere die manuellen Fähigkeiten jeder Assistenzärztin und jedes Assistenzarztes einheitlich, objektiv und transparent überprüfen zu können. In naher Zukunft wollen Thomasin und sein Team das bestehende Konzept evaluieren und weitere EPAs und Kompetenzlevels definieren. Langfristiges Ziel der Winterthurer Anästhesisten sind eine Vernetzung mit anderen Weiterbildungsstätten und ein durchgehendes Curriculum. Dies hätte auch den Vorteil, dass nicht nur wie bisher eine mündliche und schriftliche Prüfung, sondern zusätzlich die Beurteilung der praktischen Fähigkeiten über die ganze Weiterbildungszeit zum Erlangen des Facharzttitels beitragen würden.

\section{Selbstkritische Einschätzung}

Welche Kompetenzen brauchen Assistentinnen und Assistenten im ersten Jahr Chirurgie? Dieser Frage gingen die Chirurgen des Kantonsspitals Graubünden (KSGR) nach. Schliesslich steht die Implementierung eines neuen Curriculums zur Erlangung des Facharzttitels Chirurgie unmittelbar bevor. Damit verbunden ist auch die Einführung von EPAs. Um diese optimal

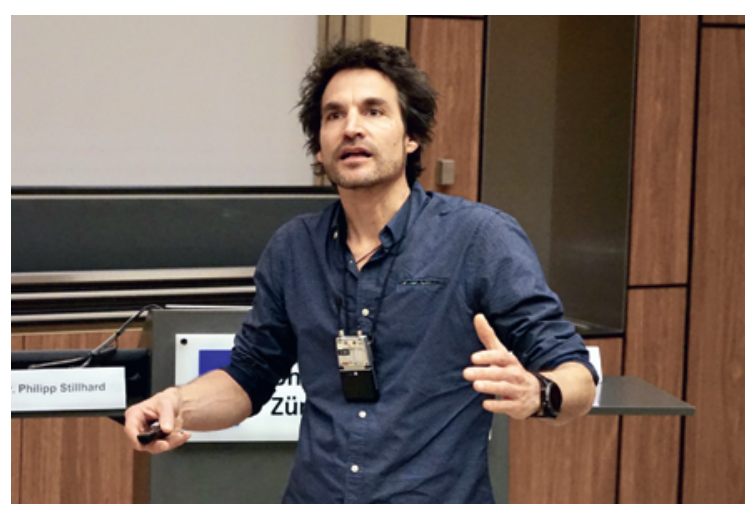

Philipp Stillhard, Chirurgie, Kantonsspital Graubünden.

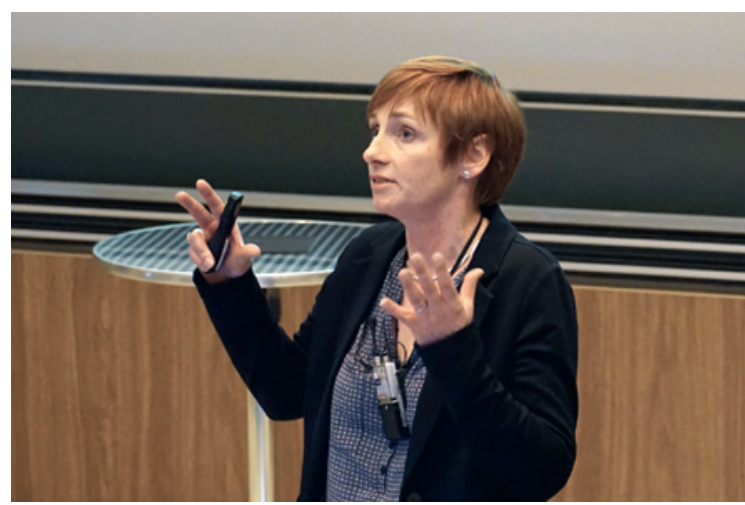

Mirjam Schuler Barazzoni, Pädiatrie, CHUV.

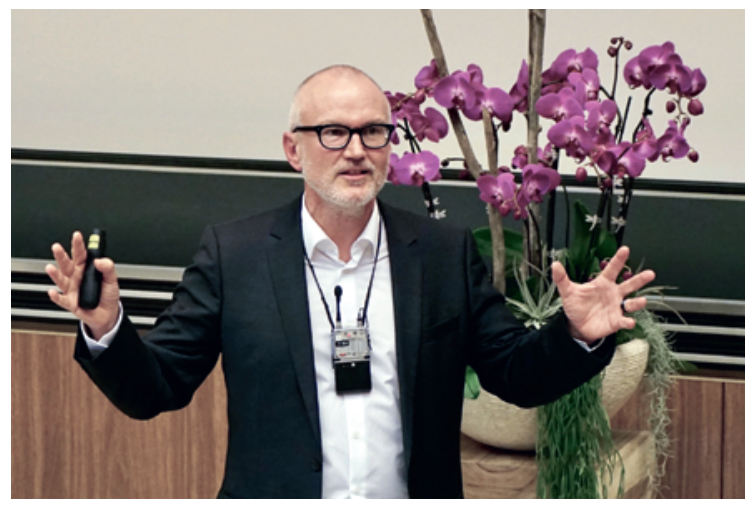

Longitudinales Curriculum für Notfallmediziner:

Roland Bingisser (USB) berichtet von seinen Erfahrungen.

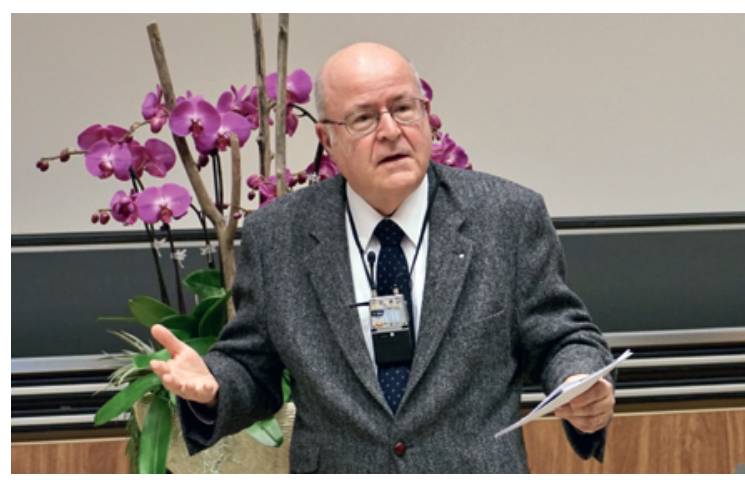

Leitung des Podiumsgesprächs: Werner Bauer,

Präsident SIWF. 
definieren zu können, befragten die Churer Chirurgen ihre Weiterzubildenden, aber auch die Weiterbildner, welche Tätigkeiten im ersten Assistenzjahr ihrer Meinung nach am wichtigsten sind. Die Resultate der Umfrage präsentierte Philipp Stillhard vom Departement für Chirurgie des KSGR. Tatsächlich konnte bei der Definition von wichtigen Tätigkeiten eine hohe Übereinstimmung zwischen den Weiterbildnern und den jungen Ärztinnen und Ärzten festgestellt werden. Eine grössere Divergenz bestand hingegen bei der Frage bezüglich der Zufriedenheit mit der Supervision hinsichtlich der Kompetenzsteigerung in jeder der CanMed-Rollen. Mit anderen Worten: Etliche Assistenten bewerteten ihre Supervision schlechter, als von der Departementsleitung erwartet. Stillhard und seine Kolleginnen und Kollegen ziehen aus diesem Resultat den Schluss, dem Aspekt der Vermittlung von Lerninhalten vermehrt Beachtung zu schenken - schliesslich korreliert der Grad der erworbenen Kompetenzen mit dem der Supervision.

\section{Longitudinales Curriculum ist machbar}

Bereits einen guten Schritt weiter sind die Notfallmediziner im Kanton Basel-Stadt. Roland Bingisser, Chefarzt am Notfallzentrum des Universitätsspitals Basel, präsentierte die Entwicklung eines longitudinalen Curriculums vom Medizinstudium bis zum

In Basel profitieren bereits die Medizinstudierenden von notfallmedizinischen Lerninhalten.

Abschluss der Weiterbildungsjahre im Bereich der Notfallmedizin. So profitieren in Basel bereits die Medizinstudierenden von notfallmedizinischen Lerninhalten. Dies mit dem Ziel, dass jeder Studienabgän- ger fähig ist, kritische Situationen zu erkennen und schnell und kompetent zu handeln. Für Bingisser steht fest, dass eine Verschränkung der Lerninhalte in der Ausbildung mit denen in der Weiterbildung zukunftsträchtig ist und, unabhängig von der gewählten Fachrichtung, den Start in den Klinikalltag erleichtert.

\section{Neue Impulse aufnehmen}

Mirjam Schuler Barazzoni vom Service de Néonantologie des CHUV und der Unité de Pédagogie Médicale der Universität Lausanne betonte in ihrem Referat, wie wichtig es sei, von der Fragmentierung wegzukommen und umfassend zu planen - das kompetenzorientierte Lernen als Kontinuum von der Aus-, über die Weiterbis in die Fortbildung. Schuler Barazzoni erinnerte auch daran, dass ein Arzt ein Arzt sei und nicht die Addition von Checklisten.

Nach diesen vertieften Einblicken in die einzelnen Projekte erörterten die Referenten in einer Podiumsdiskussion abschliessend weitere Punkte rund um die Einführung junger Ärztinnen und Ärzte in die verschiedenen Fachrichtungen.

Es ist zu hoffen, dass auch nicht anwesende Weiterbildner die neuen Impulse aufnehmen und dass die Spitalträger bzw. Spitaldirektionen trotz finanzieller Einschränkungen die ärztliche Weiterbildung weiterhin hoch gewichten und für zukunftsträchtige Neuerungen offen sind.

Bildnachweis

Matthias Scholer

\section{Podcasts und Tagungsunterlagen}

Die Podcasts und Tagungsunterlagen zum Symposium sind auf www.usz.ch/epa abrufbar. Das nächste Symposium für ärztliche Weiterbildner wird am 9. Februar 2018 stattfinden. 